\title{
Cell Death During Gangliogenesis in the Leech: Competition Leading to the Death of PMS Neurons Has Both Random and Nonrandom Components
}

\author{
E. R. Macagno and R. R. Stewart ${ }^{a}$ \\ Department of Biological Sciences, Columbia University, New York, New York 10027
}

The posteromedial, serotonin-containing (PMS) neurons are found in the ventral aspect of certain anterior segmental ganglia of adult leeches. With one exception, these cells are unpaired in all the ganglia where they are found. During early embryogenesis in Hirudo medicinalis, however, a bilateral pair of PMS neurons appears and differentiates in each of the 21 segmental ganglia (SG1-SG21). Over the next several days, one of the pair dies in SG3-SG21. Examination of the PMS neuron in any one of these segments reveals that either the right or the left cell remains, with equal probability, suggesting that the elimination of one of the pair is a random process. When unpaired PMS neurons are examined in pairs of adjacent ganglia, however, the cells are from opposite sides in the majority of cases $(\sim 88 \%)$. This observation implies that the death of a PMS neuron in 1 ganglion strongly biases which member of the pair of PMS neurons degenerates in adjacent ganglia. Detailed examination of the sequence of degeneration shows that it begins at several separate loci in the nerve cord. We propose that the mechanism responsible for the death of one of the pair of PMS neurons in a segmental ganglion is competition between these 2 cells for some trophic factor, but that the outcome of this competition is predetermined if one of the PMS neurons in an adjacent ganglion has already begun to degenerate.

Competition for a necessary factor appears to be one of the important mechanisms leading to the reduction in size of a neuron pool by cell death during neurogenesis of both vertebrates and invertebrates (Hamburger and Oppenheim, 1982; Cowan et al., 1984; Truman, 1984; Purves and Lichtman, 1985). A neuron is presumed to obtain such factors through interactions with its inputs (e.g., the laminar cells in Daphnia; Macagno, 1979) or from interactions with its targets (e.g., vertebrate motor neurons; Hamburger and Oppenheim, 1982). Alternatively, a secreted factor that acts at a distance from its source, such as nerve growth factor, may play a critical role (e.g., Hamburger et al., 1981). Implicit in the concept of competition is the idea that the process leading to the degeneration of some cells is

\footnotetext{
Received Oct. 2, 1986; revised Dec. 17, 1986; accepted Dec. 19, 1986.

We thank Darcy Kelley, Alex Peinado, Wei-Qiang Gao, and Bill Walthall for their critical readings of the manuscript, Wei-Qiang Gao and Howard Lederman for help with the preparation of specimens, and Nicholas Necles for help with the photography. This work was supported in part by NIH Grant NS-20336.

Correspondence should be addressed to E. R. Macagno, 1003B Fairchild, Columbia University, New York, NY 10027.

a Present address: Department of Pharmacology, Biocenter, Basel 4056, Switzerland.

Copyright (C) 1987 Society for Neuroscience $0270-6474 / 87 / 061911-08 \$ 02.00 / 0$
}

statistical rather than determinative, and, therefore, that the identity of those cells within the pool that will die cannot be predicted a priori. As a corollary, then, we would expect this process to depend upon one or more random variables. One such variable, for example, could be the relative order of arrival at the target of the axonal projections from the neuron pool. Another variable could be the distribution of the necessary factor, whose local availability might vary stochastically. However, the inability to predict a priori which neurons among a group will die does not mean that a random variable is necessarily part of the mechanism of competition. The inability to predict in some cases may be a consequence of the fact that neurons in a pool (e.g., motor neurons in mammals) cannot at present be assigned individual identities. Furthermore, ignorance of the details of what may be a complex process can also preclude predictability and thus give a false impression of a stochastic component in the mechanism that leads to natural cell death.

We have explored the above issues in the case of the death of specific, identified neurons, the posteromedial, serotonincontaining (PMS) neurons, in the nerve cord of the leech Hirudo medicinalis. These neurons are found in the ventral aspect of several segmental ganglia (Rude, 1969; Lent, 1982). In adult animals raised in our laboratory, PMS neurons are found cither as a pair or as single cells in the second segmental ganglion (SG2) and as an unpaired cell in SG3-SG7. As we report here, however, a bilateral pair of PMS neurons appears and differentiates in all 21 segmental ganglia early in embryogenesis. Subsequently, 1 of the pair degenerates in SG3-SG21, the surviving PMS neuron being either from the left or the right side. Postembryonically, no PMS neurons are seen in SG1 or SG8-SG21 when using the techniques we have employed (see Materials and Methods). This sequence of events occurs not only in hirudinid leeches, but has also been observed in the glossiphoniid leeches (Stuart et al., 1987). The analysis of our data shows that while the degeneration of some of the PMS neurons is determined stochastically, that of others is not.

Preliminary reports of this work have been published in abstract form (Macagno et al., 1985).

\section{Materials and Methods}

Hirudo medicinalis used in these experiments were from the breeding colony maintained in our laboratory. The colony was derived from animals obtained from suppliers in France (Ricarimpex, Bordeaux) and West Germany (Blutegel Import und Versand, Recklinghausen-Sud). Breeding and maintenance procedures were as described in the following report (Stewart et al., 1987). In brief, we maintained breeding animals at $24^{\circ} \mathrm{C}$. At this temperature, embryogenesis took about $30 \mathrm{~d}$. We staged embryos by counting days after a cocoon was laid. In the experiments 
described herc, we removed cmbryos from a cocoon as early as at $7 \mathrm{~d}$, when only approximately the anterior half of the germinal plate had recognizable neuromeres. We maintained embryos removed from their cocoon in sterile, dilute, artificial seawater until they were at the required stage of development.

Hirudo segmental ganglia can have 2, 1, or no PMS neurons, depending on their position along the ventral nerve cord. The PMS neurons are derived from left-side or right-side precursors and can, therefore, be assigned a side of origin. For technical reasons, we had to use several methods to assess the distribution of PMS neurons and their side of origin. Some methods, such as antibody labeling, worked better with embryos than with adults, whereas glyoxylic acid treatment was exactly the opposite. Determining the side of origin required that we be able to visualize the path of the main process of a PMS neuron in the neuropil, since the position of the cell body itsclf was often ambiguous (i.e., the cell body straddled the midplane of the ganglion or had even drifted to the opposite side).

Antibody labeling of embryos. Embryos were processed with rabbit antisera raised against serotonin in order to visualize the PMS neurons. Two different antisera were used. One was the kind gift of Dr. Jean Lauder. The other was obtained commercially (Immunotech). The 2 antisera yielded very similar results. Embryos were fixed overnight at $4^{\circ} \mathrm{C}$ in $4 \%$ paraformaldehyde, $0.1 \%$ picric acid in $0.1 \mathrm{M}$ sodium phosphate buffer ( $\mathrm{pH} 7.4$ ), and then rinsed in PBS ( $\mathrm{pH} 7.4$ ) containing $0.01 \%$ sodium azide twice daily for $1-2 \mathrm{~d}$ to remove the fixative thoroughly. They were then treated with trypsin $(0.6 \mathrm{mg} / \mathrm{ml}$ in PBS; BoehringerMannheim) to facilitate antibody penetration, and incubated overnight at $4^{\circ} \mathrm{C}$ in anti-serotonin at dilutions of $1: 500-1: 10,000$ in PBS containing $0.2 \%$ Triton X-100 (Sigma). After two 15 min rinses in PBS, the embryos were incubated in rhodamine-conjugated secondary antibody (goat antirabbit IgG; Boehringer-Mannheim) for $1-2 \mathrm{hr}$ at room temperature or overnight at $4^{\circ} \mathrm{C}$. Some of the embryos were processed further by rinsing with PBS (two 15 min rinses) and then adding $1 \%$ peroxidase-antiperoxidase (Accurate Chemicals) in PBS with $0.2 \%$ Triton X-100. We used $3^{\prime}-3^{\prime}$-diaminobenzidine $(4 \mathrm{mg} / \mathrm{ml})$ as the chromogen. In the majority of cases, these procedures permitted the visualization of PMS neuron cell bodies, as well as of the primary processes as they traveled into the neuropil and across posterior commissures to the contralateral side of the ganglion, allowing us to assign a left- or right-side identity to these neurons.

Visualization of serotonin-containing cells in late embryos, juveniles and adults. Since we found that antibody staining worked well only in younger embryos, we used 2 other histological techniques to study the distribution of PMS neurons in the segmental ganglia of late embryos, juveniles, and adults: glyoxylic acid-induced serotonin fluorescence (Stuart, 1981; Lent, 1982) and neutral red vital staining (Stuart et al., 1974), followed by dye-filling of individual PMS neurons. For glyoxylic acid treatment, we dissected out whole ventral nerve cords of late embryos (28-30 d), juveniles, and adults, pinned them in Sylgard-covered dishes, and incubated them for a few min in a solution containing 10 $\mathrm{mg} / \mathrm{ml}$ glyoxylic acid, $68 \mathrm{mg} / \mathrm{ml}$ sucrose, and $32 \mathrm{mg} / \mathrm{ml}$ monobasic potassium phosphate buffer, adjusted to $\mathrm{pH} 7.4$ with $\mathrm{NaOH}$ (de la Torre and Surgeon, 1976). The tissues were then air-dried for 20-30 min, placed on a slide with a few drops of mincral oil, covered with cover glass, and heated for $3-5 \mathrm{~min}$ in a $95^{\circ} \mathrm{C}$ oven. The preparations were then studied with a Leitz microscope equipped with an E-filter set for FITC epifluorescence. In successful preparations, the principal process of a PMS neuron could be observed, along with its cell body.

Vital staining with neutral red was carried out only in adult ganglia. Nerve cords were dissected out and pinned in Sylgard-covered dishes in which they were incubated in a $1 \mu \mathrm{g} / \mathrm{ml}$ solution of the dye in leech Ringer's solution (Stuart et al., 1974) for 10-20 min. They were then rinsed in fresh Ringer's solution and examined under a dissecting microscope to ascertain the number and position of PMS cell bodies stained red by the dye. Since processes are not visibly stained by this procedure, we pressure-injected cells with either the fluorescent dye Lucifer yellow (Sigma) or with HRP (Sigma Type VI) using beveled microelectrodes. The procedures used were standard and are describcd in detail in previous publications (DeRiemer and Macagno, 1981; Muller et al., 1981). Ganglia with cells filled with the fluorescent dye were mounted directly in glycerol and the geometry of the dye-filled cells assessed under epifluorescence. Ganglia with cells filled with HRP were fixed before dehydration and mounting in Permount (Fisher). Preparations viewed with epifluorescence or bright-field illumination were photographed with Kodak Tri-X film and Kodak Technical Pan 2415 film, respectively.

\section{Results}

\section{Adult distribution of PMS neurons in segmental ganglia}

Several different serotonin-containing neurons are present in the segmental ganglia of adult IIirudo (Rude, 1969; Lent, 1982). These do not appear in all ganglia uniformly. Among those that are always bilaterally paired, the Retzius, dorsolateral, and ventrolateral neurons are found in SG1-SG21, but the anteromedial cells (also called E cells; Lent, 1982) are found only in SG1SG3 (Rude, 1969). The PMS neurons in adult leeches raised in our laboratory appear as a pair or as a single cell in SG2 and as a single cell in SG3-SG7. (In a few animals out of nearly 100 that we have examined, we also found a pair of PMS cells in SG1 or an unpaired PMS cell in SG8.) This spatial distribution of PMS neurons is found not only in adults, but in juveniles and in some late embryos, just before they emerge from the egg case.

We determined the frequencies with which PMS neurons in SG2 appeared as a pair or as a single cell in 45 animals. We found 27 cases $(60 \%)$ with 2 PMS neurons and 18 cases $(40 \%)$ with an unpaired cell. (Our obscrvations differ from those of Rude (1969), who reported finding a pair of PMS neurons in SG 1 and a single cell in SG2-SG21 in adults of the same species.)

To examine their anatomy and projections, we filled a total of 15 PMS neurons in different adult ganglia with HRP. All cells were seen to extend 1 principal process from the soma, which traveled a short distance anteriorly in the ipsilateral side of the neuropil, decussated through a posterior commissure, and then traveled again anteriorly, exiting the ganglion along the contralateral connective nerve and projecting to adjacent, more rostral, ganglia (Fig. 1). No projection to more posterior ganglia was seen. PMS cells in SG2 projected to and through SG1 and continued through the subesophageal ganglion to the circumesophageal connective (they may have traveled further, but we were not able to inject enough dye to examine the complete projection, since these cells have relatively small somata). PM ncurons in SG3-SG7 projected at least as far as the adjacent, more rostral, ganglion. Within the ganglion of origin, there was extensive bilateral arborization in the posterior half of the neuropil, whereas the arborization in the anterior half was limited to a medial region. The extent of the arborization varied from segment to segment (Fig. 1). Because insufficient dye was injected, we were not able to determine from our dye fills whether the projections to adjacent ganglia also arborized in their ncuropils.

From the examination of the position of the cell body or of the path followed by its principal process (i.e., the direction of decussation), we were able to assign a side of origin to 186 out of 211 unpaired PMS neurons, identified in 45 preparations, that showed adult distribution of PMS neurons (Table 1). Among these, 96 were left-side and 90 were right-side PMS neurons. This near equality in the number of left and right cells was also true for individual segmental ganglia (Table 1), indicating that in any adult Hirudo in our colony, the probability of finding a right PMS neuron in any particular ganglion (among those having unpaired PMS neurons) was $\sim 0.5$.

When we considered the sides of origin of 2 unpaired PMS neurons in adjacent ganglia, however, we found that the cells were from opposite sides in 138 cases $(\sim 91 \%)$ and from the same side in only $13(\sim 9 \%)$. Thus, alternating sides from ganglion to ganglion appears to be the predominant pattern for 

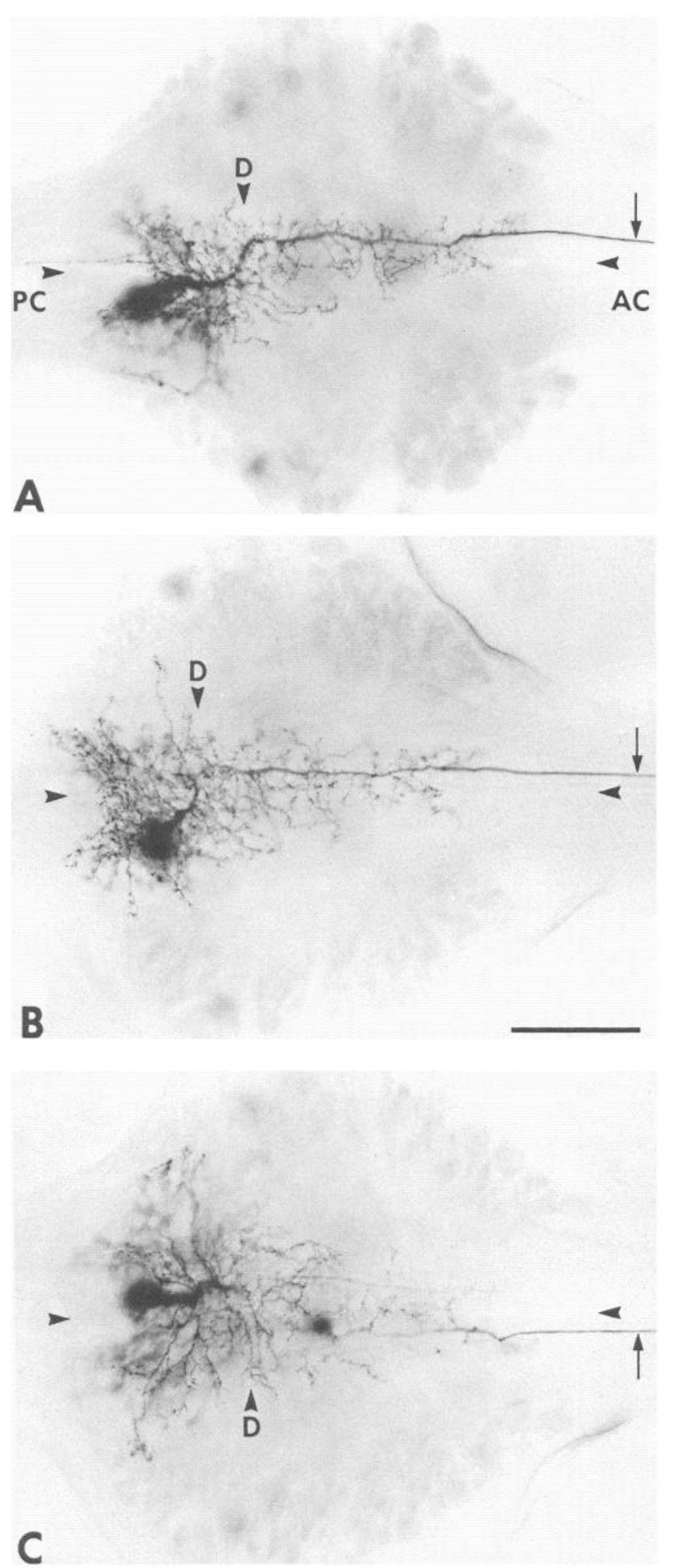

Figure 1. Photomicrographs of whole mounts of adult PMS neurons filled with HRP. All 3 neurons are from a single specimen. $A$, One of a pair of PMS neurons found in SG2 (the other was not filled with dye). $B$, PMS neuron in SG3. C, PMS neuron in SG4. All 3 neurons decussate and project anteriorly (arrows indicate these axons) to neighboring ganglia, but the extent of secondary branching is significantly greater in SG4 than in the other 2 ganglia. The branching is distributed bilaterally in a more symmetric manner in SG3 and SG4, which had single PMS neurons, than in SG2, which in this case had 2 PMS neurons. No projections to the periphery are made by these neurons, suggesting that
Table 1. Numbers of left and right unpaired PMS neurons in late embryos, juveniles, and adults (44 specimens) ${ }^{a}$

\begin{tabular}{llll} 
& \multicolumn{2}{l}{$\begin{array}{l}\text { Side of } \\
\text { unpaired PMS neuron }\end{array}$} \\
\cline { 2 - 4 } Segmental ganglion & Left & Right & Unknown \\
\hline SG2 & 7 & 10 & 1 \\
SG3 & 19 & 21 & 4 \\
SG4 & 21 & 18 & 5 \\
SG5 & 17 & 18 & 6 \\
SG6 & 19 & 16 & 6 \\
SG7 & 12 & 15 & 3 \\
SG8 & 1 & 1 & 0 \\
Total & 96 & 90 & 25 \\
\hline
\end{tabular}

${ }^{a}$ Not all ganglia were analyzed in every specimen.

unpaired adult PMS neurons. We have called this feature the "alternate-side rule."

\section{Initial labeling of PMS neurons with antisera}

Embryonic development in Hirudo takes approximately $30 \mathrm{~d}$ at $\sim 24^{\circ} \mathrm{C}$. Labeling of embryos at several stages (from 7 to 21 d) of development with the antisera raised against serotonin revealed that the Retzius cells are the first neurons to express detectable levels of serotonin. We saw labeled Retzius cells in anterior ganglia as early as at $7.5 \mathrm{~d}$. Labeling of the other serotonin-containing cells lagged behind, with the PMS neurons being the last to label in any one ganglion. We first detected labeling of the PMS neurons in anterior ganglia of 9-d-old animals. In 3 specimens from 1 clutch, we saw stained PMS neurons in SG1-SG7, 8 or 9 (the most posterior SG containing labeled cells varied from animal to animal). Most ganglia at this stage contained 2 such cells. With time, labeled PMS neurons appeared in increasingly more posterior ganglia and, by $11 \mathrm{~d}$, pairs of PMS cells could be seen in each of the 21 segmental ganglia. Examination at high magnification revealed that the PMS neurons had, by $11 \mathrm{~d}$, extended their primary processes into the neuropil. In most cases these processes could be followed only to their decussations; beyond that, they joined tracts with many other antibody-labeled neurites, from which they could not be distinguished with the light microscope. We were not able to inject a dye into these cells at these early stages of development because they could not be identified in the live preparations.

\section{Degeneration of PMS neurons between 12 and $21 d$ of embryogenesis}

We examined 28 embryos that were labeled with antisera raised against serotonin. We found PMS neurons in several stages of degeneration in embryos 12-14 d of age (Fig. 2). Degeneration of these neurons followed a sequence similar to the one we have described for the bipolar cells (Stewart et al., 1987). The cells first lost their processes, and the intensity with which their so-

\section{$\leftarrow$}

they are interneurons. The dorsal aspect of the ganglia is towards the viewer, anterior is to the right, posterior to the left. $A C$, Anterior connective nerve; $P C$, posterior connective nerve; $D$, decussation; arrowheads, midplane of the ganglia. Bar, $100 \mu \mathrm{m}$. 

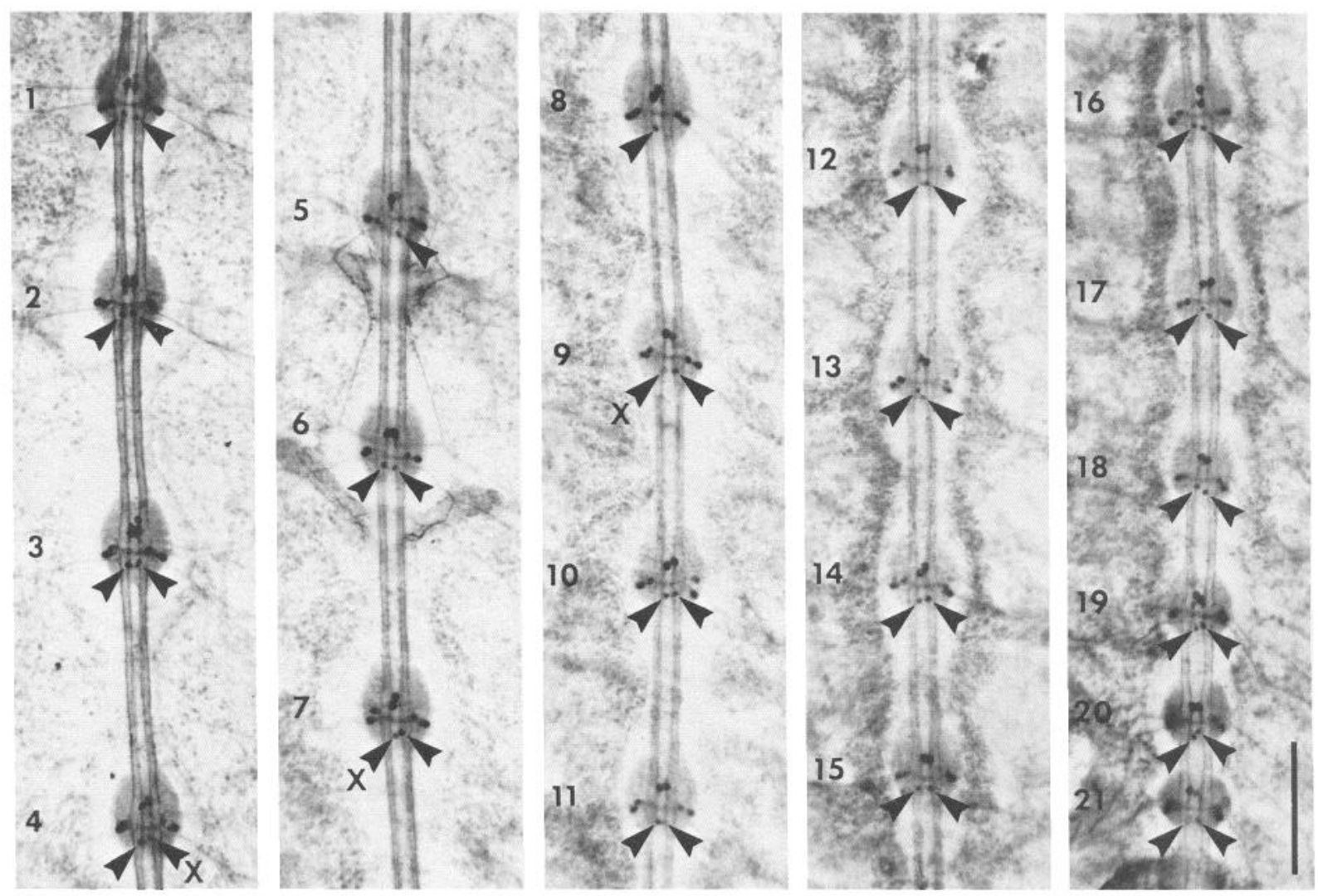

Figure 2. Low-power photomicrographs of a 12-d-old leech embryo labeled with anti-serotonin antibodies. This specimen is the same as the "b" specimen in Table 2. The 21 segmental ganglia are numbered on the left. Arrowheads point at the PMS neurons in each ganglion; the arrowheads with $a \times$ indicate cells that are degenerating (i.e., they lack processes and cell bodies are fragmenting). Two cells, the left PMS neuron in SG5 and the right one in SG8, are missing and presumed dead, since pairs of PMS neurons are present in these ganglia in embryos 1-2 d younger. The quality of staining varies slightly from segment to segment, and even within 1 segment, so that in some cases apparently healthy PMS neurons are more lightly stained than their neighbors. Bar, $200 \mu \mathrm{m}$.

mata labeled with the antibody decreased. They then became fragmented into small pieces, which could be identified because they retained anti-serotonin immunoreactivity (Fig. 3). Finally, the fragments themselves disappeared, presumably as they were phagocytosed by glia or other cells. No traces of degenerating PMS neurons remained in 15-d or older embryos. Examination of sectioned ganglia in embryos of these ages revealed the existence of pycnotic fragments in the region where the PMS cells degenerated (Stewart et al., 1986).

The degeneration of the PMS neurons in SG3-SG21 occurred within days 12 to 14 of embryogenesis, but not with a set spatial pattern. At 12-13 d, cells began to degenerate at about the same time in several segments along the nerve cord (Fig. 2). The time of degeneration of cells in any given segment varied from animal to animal (see Table 2). Further, cells on the right side died with a frequency equal to that of those on the left, as we would expect from the observations on adults presented above. The unpaired PMS neurons retained detectable levels of serotonin-like immunoreactivity in SG8-SG21 until at least day 21 (see Table 3).

When we examined the right- or left-side identities of unpaired PMS neurons in 2 adjacent ganglia in 12-21 d embryos, we obtained results similar to those for older animals. Of 183 pairs of ganglia for which the location of the PMS neurons could be assigned unambiguously, 161 pairs $(\sim 88 \%)$ showed cells on opposite sides, while only 22 pairs $(\sim 12 \%)$ showed both cells on the same side. In 68 other cases, either 1 or both of the cells could not be assigned to either the left or the right side of the ganglion. Thus, the alternate-side rule holds with nearly the same accuracy in SG3-SG21 of middle-stage embryos as it does in SG2-SG7 of adults.

At some time between day 21 and the end of embryogenesis, the pair of PMS neurons in SG1 and the unpaired ones in SG8SG21 cease being visible in some animals. In most cases, this happens postembryonically. Since we did not study these stages in this series of experiments, we cannot specify whether these PMS neurons actually degenerate or stop containing serotonin. Either of these possibilities would preclude our finding them in those segments in older animals using the techniques we employed.

\section{Discussion}

Generation and degeneration of PMS neurons

PMS neurons appear and differentiate as a bilateral pair in all 21 segmental ganglia (SG1-SG21) of the Hirudo ventral nerve cord between 9 and $11 \mathrm{~d}$ of embryogenesis, earlier in anterior segments and later in posterior ones. Degeneration begins at 12 d; by day 15 only a single PMS neuron can be found in SG3SG21. In SG2, a PMS neuron degenerated in only about half the cases studied, 2 cells remaining in the others - a situation we also find in adults. During the middle period of embryogenesis, from about 10 to $20 \mathrm{~d}$, the number of cells in the segmental ganglia of hirudiniid leeches decreases by 10-20\% (Stewart et al., 1986; C. Baptista and E. R. Macagno, unpublished obser- 

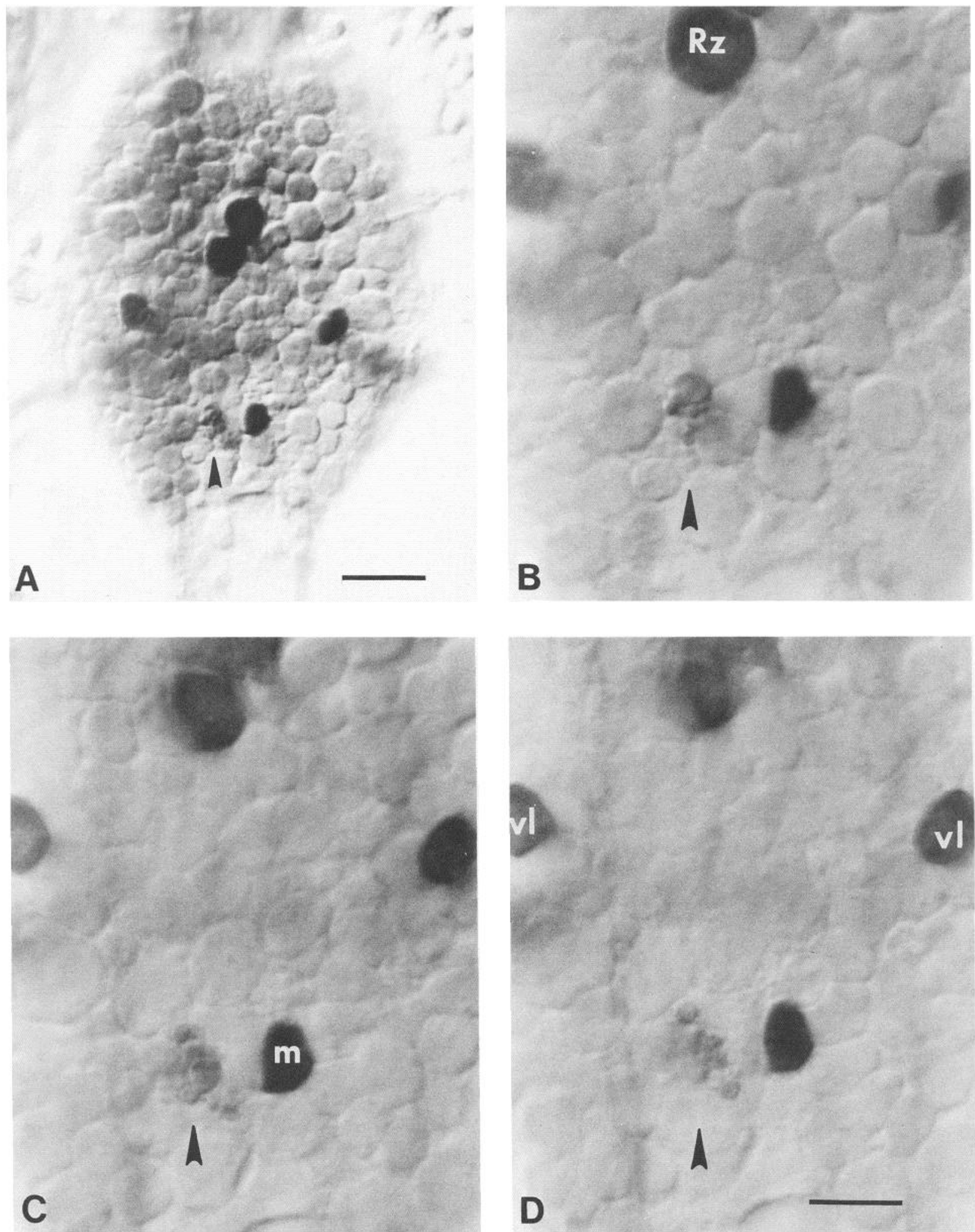

Figure 3. Degeneration of the right PMS neuron in SG7 of a $12 \mathrm{~d}$ embryo as shown by labeling with anti-serotonin antibody. A, Low-power micrograph of SG7 showing labeled serotonin-containing neurons. Arrowhead points towards the degenerating PMS neuron, which still remains immunoreactive. Eventually, as seen in other preparations, no trace of the labeled, dying PMS neurons can be found. $B-D$, Higher-magnification micrographs of the PMS neurons in $A$ are shown at 3 different focal planes (ventral to dorsal). Arrowheads point to the dying PMS neuron. $B$, Cell labeled $R z$ is 1 of the 2 Retzius neurons. $C$, PMS neuron on the right side is indicated by the letter $m$. $D$, Pair of ventrolateral neurons is indicated by the letters $v l$. Anterior is towards the top. Bar in $A, 20 \mu \mathrm{m}$; bar in $D, 10 \mu \mathrm{m}$ (applies to $B-D$ ). 
Table 2. PMS neurons in 6 embryos $12-13 \mathrm{~d}$ old

\begin{tabular}{|c|c|c|c|c|c|c|}
\hline \multirow[b]{2}{*}{$\begin{array}{l}\text { Segmental } \\
\text { ganglion }\end{array}$} & \multicolumn{6}{|c|}{ Specimen } \\
\hline & $\frac{\mathrm{a}}{\mathrm{Ir}}$ & $\frac{b}{1 r}$ & $\frac{\mathrm{c}}{\mathrm{lr}}$ & $\frac{\mathrm{d}}{1 \mathrm{r}}$ & $\frac{\mathrm{e}}{1 \mathrm{r}}$ & $\frac{f}{1 r}$ \\
\hline SG1 & $\infty$ & $\infty$ & $\infty$ & $\infty$ & $\infty$ & $\infty$ \\
\hline SG2 & $\infty$ & $\infty$ & $\infty$ & $\infty$ & $\infty$ & $\infty$ \\
\hline SG3 & $\infty$ & $\infty$ & $\infty$ & $\infty$ & ox & $\infty$ \\
\hline SG4 & $\infty$ & OX & $\times 0$ & $\infty$ & $x_{0}$ & $\infty$ \\
\hline SG5 & $\infty$ & $\infty$ & $\infty$ & ox & $O X$ & $\infty$ \\
\hline SG6 & $\times 0$ & $\infty$ & $\infty$ & xo & $\infty$ & $\infty$ \\
\hline SG7 & $\infty$ & $x 0$ & $\infty$ & $x_{0}$ & xo & $\infty$ \\
\hline SG8 & $\infty$ & $\infty$ & $\infty$ & ox & OX & $\infty$ \\
\hline SG9 & $\infty$ & $x_{0}$ & $\infty$ & $x_{0}$ & $x_{0}$ & - \\
\hline SG10 & $\infty$ & $\infty$ & $\infty$ & $\infty$ & $\infty$ & 0 \\
\hline SG11 & $\infty$ & $\infty$ & $\infty$ & $x_{0}$ & $\infty$ & - \\
\hline SG12 & - & $\infty$ & $\oplus$ & ox & $\infty$ & - \\
\hline SG13 & $\infty$ & $\infty$ & $\infty$ & ox & $\infty$ & $\infty$ \\
\hline SG14 & $\infty$ & $\infty$ & $\infty$ & $\infty$ & $\infty$ & 0 \\
\hline SG15 & $x_{0}$ & $\infty$ & $O x$ & $\infty$ & ox & $\infty$ \\
\hline SG16 & ox & $\infty$ & $\infty$ & $\infty$ & $\times 0$ & $\infty$ \\
\hline SG17 & $\infty$ & $\infty$ & $\infty$ & $\infty$ & $\infty$ & $\infty$ \\
\hline SG18 & $\infty$ & $\infty$ & $\infty$ & $\infty$ & $\times 0$ & $\infty$ \\
\hline SG19 & $\infty$ & $\infty$ & $\infty$ & $\infty$ & $O X$ & $\infty$ \\
\hline SG20 & $\infty$ & $\infty$ & $\infty$ & $\infty$ & $x_{0}$ & $\infty$ \\
\hline SG21 & $\infty$ & $\infty$ & $\infty$ & - & $\infty$ & - \\
\hline
\end{tabular}

$\mathrm{l}, \mathrm{r}$, Left and right sides of the segmental ganglia. $O$, Healthy PMS neuron; if located in the middle, it means we were unable to assign a side. PMS neuron missing. $X$, Dying PMS neuron. - , No PMS neurons visible because of poor staining.

vations). The data on the degeneration of the PMS neurons, along with our observations on the death of another identified leech cell (the bipolar cell; Stewart et al., 1987), show that at least part of this decrease in cell number is due to the degeneration of differentiated cells.

Degeneration begins with the loss of processes and culminates in the fragmentation of the cell bodies before they entirely disappear. The fragments can be easily identified by location and by their retention of serotonin-like immunoreactivity. The same sequence takes place in the degeneration of the bipolar cells (Stewart et al., 1987; these cells do not contain serotonin at levels detectable by our methods), suggesting that this may be a common pathway for the removal of differentiated cells in the leech. Cells that disintegrate as they die have also been observed in insect development [e.g., the D-IV motoneurons of Manduca (Truman and Schwartz, 1982), neurons Q1 and Q2 (Raper et al., 1983) and the muscle pioneer cell d133a (Ball et al., 1985) of grasshoppers].

Some very late embryos (28-30 d old), as well as juveniles and adults from our Hirudo colony, do not have PMS neurons in SG1 or in SG8-SG21 (when stained either by the glyoxylic acid treatment or with neutral red). Since PMS neurons are still present in these ganglia at $21 \mathrm{~d}$, it follows that they disappear between 21 and $28 \mathrm{~d}$ of development. Our observations do not permit us to specify whether they die or lose detectable levels of serotonin-like immunoreactivity.

The distribution of PMS ncurons in lecches from a different family, the Glossiphoniidae (Mann, 1962) differs somewhat from that in leeches of the family Hirudidae, to which Hirudo medicinalis belongs. In the glossiphoniids, unpaired PMS neurons
Table 3. PMS neurons in 6 embryos 18-21 $\mathrm{d}$ old

\begin{tabular}{|c|c|c|c|c|c|c|}
\hline \multirow[b]{2}{*}{$\begin{array}{l}\text { Segmental } \\
\text { ganglion }\end{array}$} & \multicolumn{6}{|c|}{ Specimen } \\
\hline & $\frac{18 \mathrm{a}}{1 \mathrm{r}}$ & $\frac{19 \mathrm{a}}{1 \mathrm{r}}$ & $\frac{20 \mathrm{a}}{1 \mathrm{r}}$ & $\frac{20 b}{1 r}$ & $\frac{20 \mathrm{c}}{1 \mathrm{r}}$ & $\frac{21 \mathrm{a}}{1 \mathrm{r}}$ \\
\hline SG1 & $\infty$ & $\infty$ & $\oplus$ & $\infty$ & - & $\infty$ \\
\hline $\mathrm{SG} 2$ & $\infty$ & $\infty$ & $\infty$ & $\infty$ & $\infty$ & $\infty$ \\
\hline SG3 & $\infty$ & $\infty$ & $\infty$ & $\infty$ & $\infty$ & $\infty$ \\
\hline SG4 & $\infty$ & 0 & $\infty$ & $\infty$ & $\infty$ & $\infty$ \\
\hline SG5 & $\infty$ & $\infty$ & $\infty$ & $\infty$ & $\infty$ & $\infty$ \\
\hline SG6 & $\oplus$ & $\oplus$ & $\oplus$ & $\infty$ & $\infty$ & $\infty$ \\
\hline SG7 & $\infty$ & $\infty$ & - & $\infty$ & $\infty$ & $\infty$ \\
\hline SG8 & $\infty$ & $\infty$ & - & $\infty$ & $\infty$ & $\infty$ \\
\hline SG9 & $\infty$ & 0 & $\infty$ & $\infty$ & $\infty$ & $\infty$ \\
\hline SG10 & $\bullet$ & 0 & $\infty$ & $\infty$ & $\infty$ & $\infty$ \\
\hline SG11 & $\infty$ & $\infty$ & or & 0 & $\infty$ & $\infty$ \\
\hline SG12 & $\infty$ & O & $\infty$ & 0 & 0 & $\infty$ \\
\hline SG13 & $\infty$ & 0 & 0 & 0 & $\infty$ & $\infty$ \\
\hline SG14 & $\infty$ & $\infty$ & O & O & $\infty$ & $\infty$ \\
\hline SG15 & $\infty$ & $\infty$ & $\infty$ & 0 & $\infty$ & $\infty$ \\
\hline SG16 & $\infty$ & $\infty$ & $\infty$ & $\infty$ & $\infty$ & $\infty$ \\
\hline SG17 & $\infty$ & $\infty$ & $\infty$ & $\infty$ & $\infty$ & 0 \\
\hline SG18 & $\infty$ & 0 & $\infty$ & $\infty$ & $\infty$ & 0 \\
\hline SG19 & $\infty$ & - & $\infty$ & $\infty$ & $\infty$ & $\infty$ \\
\hline $\mathrm{SG} 20$ & $\infty$ & - & $\infty$ & $\infty$ & 0 & $\infty$ \\
\hline $\mathrm{SG} 21$ & $\infty$ & - & $\infty$ & $\infty$ & 0 & 0 \\
\hline
\end{tabular}

$\mathrm{l}, \mathrm{r}$, Left and right sides of the segmental ganglia. $\mathrm{O}$, Healthy PMS neuron; if located in the middle, it means we were unable to assign a side. $\bullet$, PMS neuron missing. -, No PMS neurons visible because of poor staining.

are found in SG1-SG7 in adults, and they appear to originate from the right or left side at random. As in Hirudo, a bilateral pair of these cells appears early in development in each of these anterior ganglia and then one of them dies (Stuart et al., 1987). Unlike our findings with Hirudo, however, no PMS neurons are seen in SG8-SG2 1 at any embryonic stage, using anti-serotonin antibodies. As pointed out by Stuart and collaborators, this need not mean that there are no homologs of the PMS cells in those ganglia. It is possible that they do exist but do not contain serotonin at a level that is immunohistochemically detectable.

\section{Do PMS neurons die as a result of competition?}

Since PMS neurons differentiate (i.e., their axons are seen projecting into the neuropil) before one of the pair dies, it appears likely that cell death is the result of competition for synaptic targets and/or growth factors, as has been described for the development of the vertebrate nervous system (Hamburger and Oppenheim, 1982). The fact that the death of at least some of the PMS neurons appears to be randomly determined (see the next section) is also suggestive of a competitive interaction between the left and right PMS neurons in those ganglia where one of the 2 cells degenerates randomly. Experimental evidence in favor of competition has been provided by the studies of Stuart et al. (1987) on the PMS neurons of glossiphoniid leeches. Since the left and right PMS neurons are derived from the left and right $\mathrm{N}$ teloblasts, Stuart and collaborators were able to prevent the appearance of all the PMS ncurons on 1 side (along with all other progeny of the teloblast) by ablating the corresponding teloblast very early in development. The result of this perturbation was the survival of all the PMS neurons derived 
from the remaining $\mathrm{N}$ teloblast. Two likely conclusions can be drawn from this result: (1) The competition that usually leads to the degeneration of 1 PMS neuron is absent under these circumstances; (2) this competition is normally between the PMS neurons in the same ganglion and not between cells on the same side in adjacent ganglia (but see our discussion of this point in the following section). The only caveat to these interpretations of the outcome of these experiments is that the ablation of the $\mathrm{N}$ teloblast leads to a massive loss of cells (the $\mathrm{N}$ teloblasts give rise to about half of the neurons found in a segmental ganglion; Kramer and Weisblat, 1985), and therefore the conditions under which the daughters of the remaining $\mathrm{N}$ teloblast find themselves are quite abnormal. It is possible, for example, that there is a greater relative amount of a critical growth factor available to the remaining PMS neurons in these circumstances, allowing all on the same side to survive. Nonetheless, competition seems a strong possibility.

\section{Is the death of a particular PMS neuron randomly determined?}

The data presented in Table 1 show that, for any particular segmental ganglion with a single PMS neuron, it is nearly equally probable that the cell is a right- or a left-side cell. The apparent implication of this observation is that the mechanism responsible for eliminating one of the original pair of PMS neurons depends on some random variable that affects cells on the left and right sides equally. This conclusion, however, is not entirely correct, as is shown by an examination of the pattern of cell death along the nerve cord in any single specimen. When we examine which sides the remaining unpaired cells belong to in adjacent segments, the predominant result (in $\sim 90 \%$ of the cases) is that they belong to opposite sides. This means that, although wc cannot predict a priori which of the PMS neurons in ganglion $\mathrm{X}$ will die in specimen $\mathrm{Y}$, we can predict with $90 \%$ reliability that those that die in ganglia adjacent to $X$ will be from the other side. We interpret this feature as evidence that the death of a particular PMS neuron is not determined in a purely random manner: The mechanism must include a nonrandom component that depends on the state of PMS neurons in other ganglia.

Further insight into these events follows from a consideration of the spatial patterns of cell degeneration seen in embryos 12$13 \mathrm{~d}$ old (Table 2). We observe that PMS neurons do not all degenerate at the same time, nor do they do so in a regular caudal or rostral progression. Rather, degenerating PMS neurons are first seen in a few clusters of between 1 and 3 adjacent segmental ganglia at several (between 1 and 4) locations along the ventral nerve cord. The locations of these clusters differ among the specimens we have examined (cf. Table 2, specimens $a, b$, and c), which suggests that the segments in which the degeneration of PMS neurons first takes place are not specified. In clusters of 2 or 3 adjacent ganglia with dead or degenerating PMS neurons, the healthy PMS neurons invariably follow the alternate-side rule (see Table 2). In embryos in which more PMS neurons have died, the clusters are larger, some have apparently merged with one other, and exceptions to the alternate-side rule are observed (see Table 2, specimens $d$, e, and f).

These features of the spatiotemporal pattern of the death of PMS neurons lead us to conclude that the mechanism involved depends not only on interactions between cells in the same ganglion, but also on interactions between cells in adjacent ganglia. The interaction between cells in the same ganglion is a competition for some necessary factor, depends upon a random variable, such as the distribution of this putative factor, and gives an equal probability for survival to either of the 2 PMS neurons. The interaction between cells in adjacent ganglia becomes effective once a PMS neuron begins to degenerate in 1 ganglion, determines that the PMS neuron on the opposite side will die in the other ganglion, and does not depend on a random variable. A consequence of this second interaction is that the death of PMS neurons propagates from the segmental ganglia where degeneration first occurs, yiclding what wc have called the alternate-side rule. Since degeneration appears to begin at several separate loci randomly, we expect to find pairs of adjacent ganglia where the alternate-side rule is broken, depending upon the sides and locations of the PMS neurons that degenerate initially. As shown by dye-filling adult PMS neurons (Fig. 1), these cells project to adjacent anterior ganglia. This interganglionic projection could be the mediator of the interaction between PMS neurons in neighboring ganglia that results in the alternate-side rule.

This model for the spatiotemporal pattern of the death of PMS cells makes a prediction that can be tested with our data. The maximum number of separate clusters of degenerating PMS neurons we have observed in 12-13-d embryos is 5. If, as our data indicate, these clusters are randomly located along the cord, we would predict that $50 \%$ of the time, as cell death propagates from these clusters, we would observe an exception to the alternate-side rule, which means 2-3 exceptions per embryo. The data from embryos $18-21 \mathrm{~d}$ old (see Table 3 ) show an average of 2 exceptions per embryo, in good agreement with this prediction. Conversely, if our model is correct, an average of 2 exceptions to the alternate-side rule in the older embryos would suggest that the number of separate segments in which cell death initially occurs must be 4 , on the average. The data from 12-dold embryos show an average of 3 , rather than 4 . However, the group of 12-d embryos includes several animals with only 1 or 2 clusters of dying PMS cells; presumably, these animals could have had more clusters had they been killed a few hours later. We conclude that the number of separate clusters of dying PMS neurons and the number of exceptions to the alternate-side rule are consistent with the idea that cell death is initiated from the initial loci by following the alternate-side rule.

What is the nature of the interaction between PMS neurons in adjacent ganglia? There are several possibilities. One is that ipsilateral homologs in adjacent ganglia are also competing for some necessary factor with each other, and that the death of 1 of them, by removing that competition, gives an overwhelming advantage to the remaining one in its competition with the contralateral homolog in its own ganglion. A different possibility is that the dying cell-since its axon projects to the contralateral side of the next anterior ganglion - releases a signal that causes the death of the PMS neuron on that side. Several variations upon these 2 themes could also be proposed. However, we lack enough information at present to select among any of these possibilities.

In summary, the observations we have presented here have led us to conclude that the mechanism responsible for the death of PMS neurons in Hirudo involves interactions between the cells in the same and in adjacent ganglia. The nature of these interactions appears to be such that the process of eliminating one of the pair in each ganglion has both random and nonrandom components. 


\section{References}

Ball, E. E., R. K. Ho, and C. S. Goodman (1985) Development of neuromuscular specificity in the grasshopper embryo: Guidance of motoneuron growth cones hy muscle pioneers. J. Neurosci. 5: 18081819.

Cowan, W. M., J. W. Fawcett, D. D. M. O'Leary, and B. B. Stanfield (1984) Regressive events in neurogenesis. Science 225: 1258-1265.

de la Torre, J. C., and J. W. Surgeon (1976) A methodological approach to rapid and sensitive monoamine histofluorescence using a modified glyoxylic acid technique: The SPG method. Histochemistry 49: 8193.

DeRiemer, S. A., and E. R. Macagno (1981) Light microscopic analysis of contacts between pairs of identified leech neurons with combined use of horseradish peroxidase and Lucifer yellow. J. Neurosci. 1: 650657.

Hamburger, V., and R. W. Oppenheim (1982) Naturally occurring neuronal death in vertebrates. Neurosci. Comment. 1: 39-55.

Hamburger, V., J. K. Brunso-Bechtold, and J. Yip (1981) Neuronal death in the spinal ganglia of the chick embryo and its reduction by nerve growth factor. J. Neurosci. 1: 60-71.

Kramer, A. P., and D. A. Weisblat (1985) Developmental neural kinship groups in the leech. J. Neurosci. 5:388-407.

Lent, C. M. (1982) Fluorescent properties of monoamine neurons following glyoxylic acid treatment of intact ganglia. Histochemistry 75: 77-89.

Macagno, E. R. (1979) Cellular interactions and pattern formation in the development of the visual system of Daphnia magna (Crustacea, Branchiopoda). Dev. Biol. 73: 206-238.

Macagno, E. R., R. R. Stewart, and D. Spergel (1985) Differentiation and death of cells in the leech CNS. II. The unpaired medial serotoninergic neurons. Soc. Neurosci. Abstr. 11: 957.
Mann, K. H. (1962) Leeches (Hirudinea), pp. 22-29, Pergamon, New York.

Muller, K., J. Nicholls, and G. Stent (1981) Neurobiology of the Leech, pp. 266-271, Cold Spring Harbor Laboratory, Cold Spring Harbor, New York.

Purves, D., and I. W. Lichtman (1985) Principles of Neural Development, pp. 131-153, Sinauer, Sunderland, MA.

Raper, J. A., M. Bastiani, and C. S. Goodman (1983) Pathfinding by neuronal growth cones in grasshopper embryos I. Divergent choices made by the growth cones of sibling neurons. J. Neurosci. 3: 20-30.

Rude, S. (1969) Monoamine-containing neurons in the central nervous system and peripheral nerves of the leech, Iirudo medicinalis. J. Comp. Neurol. 136: 349-372.

Stewart, R. R., D. Spergel, and E. R. Macagno (1986) Segmental differentiation in the leech nervous system: The genesis of cell number in the segmental ganglia of Haemopis marmorata. J. Comp. Neurol. 253: 253-259.

Stewart, R. R., W.-Q. Gao, A. Peinado, B. Zipser, and E. R. Macagno (1987) Cell death during gangliogenesis in the leech: Bipolar cells appear and then degenerate in all ganglia. J. Neurosci. 7: 1919-1927.

Stuart, A. E., A. J. Hudspeth, and Z. W. Hall (1974) Vital staining of specific monoamine-containing cells in the leech nervous system. Cell Tissue Res. 153: 55-61.

Stuart, D. K. (1981) Monoamine neurons in embryonic and adult leeches. Soc. Neurosci. Abstr. 7: 2

Stuart, D. K., S. S. Blair, and D. A. Weisblat (1987) Cell lineage, cell death, and the developmental origin of identified serotonin- and dopamine-containing neurons in the leech. J. Neurosci. 7: 1107-1122.

Truman, J. W. (1984) Cell death in invertebrate nervous systems. Annu. Rev. Neurosci. 7: 171-188.

Truman, J. W., and L. M. Schwartz (1982) Insect systems for the study of programmed neuronal death. Neurosci. Comment. 1: 66-72. 